\title{
A Note on Topological Properties of Non-Hausdorff Manifolds
}

\author{
Steven L. Kent, Roy A. Mimna, and Jamal K. Tartir \\ Department of Mathematics and Statistics, Youngstown State University, One University Plaza, \\ Youngstown, $\mathrm{OH} 44555$, USA \\ Correspondence should be addressed to Jamal K. Tartir, tartir@ysu.edu
}

Received 26 September 2008; Revised 11 February 2009; Accepted 29 June 2009

Recommended by Francois Goichot

The notion of compatible apparition points is introduced for non-Hausdorff manifolds, and properties of these points are studied. It is well known that the Hausdorff property is independent of the other conditions given in the standard definition of a topological manifold. In much of literature, a topological manifold of dimension $n$ is a Hausdorff topological space which has a countable base of open sets and is locally Euclidean of dimension $n$. We begin with the definition of a non-Hausdorff topological manifold.

Copyright (C) 2009 Steven L. Kent et al. This is an open access article distributed under the Creative Commons Attribution License, which permits unrestricted use, distribution, and reproduction in any medium, provided the original work is properly cited.

\section{Topological Properties of Non-Hausdorff Manifolds}

Definition 1.1. A non-Hausdorff manifold of dimension $n$ is a topological space which has a countable base of open sets and is locally Euclidean of dimension $n$.

Since every point of a non-Hausdorff manifold has a Euclidean neighborhood, it is easy to show that every non-Hausdorff manifold is $T_{1}$.

We now briefly review some of the well-known properties of non-Hausdorff manifolds. Since $\mathbb{R}^{n}$ is locally compact, a non-Hausdorff manifold of dimension $n$ is locally compact. In some of literature, compactness is only defined in Hausdorff spaces. In such cases, compact subsets must be closed. Compact subsets of $T_{1}$-spaces, however, need not to be closed. This remains true for non-Hausdorff manifolds (Example 1.2). A non-Hausdorff manifold of dimension $n$ must be locally connected. Since a non-Hausdorff manifold $M$ has a countable base of open sets, $M$ is Lindelöf; that is, every open cover of $M$ has a countable subcover. Further, since locally compact Lindelöf spaces are sigma-compact, it follows that a non-Hausdorff manifold $M$ of dimension $\mathrm{n}$ is sigma-compact. Finally, we note that when $M$ is not Hausdorff, it is not regular.

We now consider the property of paracompactness. A Hausdorff space $X$ is paracompact if every open covering $\mathcal{U}$ of $X$ has a locally finite refinement $\mho$. That is, each 
$V \in \mathcal{U}$ is contained in some $U \in \mathcal{U}$ and each $x \in X$ has a neighborhood $N$ which meets only finitely many sets in $\mho$. Paracompactness can be defined for $T_{1}$-spaces as follows. A $T_{1}$-space $X$ is paracompact if and only if each open covering of $X$ has an open barycentric refinement, where $\mho$ is a barycentric refinement of $\mathcal{U}$ if the collection $\{\operatorname{St}(x, \mho): x \in X\}$ refines $\mathcal{U}$, where $S t(x, \mho)=\cup\{V \in U: x \in V\}$. A space is metacompact if every open cover has a point finite refinement. Since Hausdorff second countable manifolds are metrizable, they are paracompact and hence metacompact. In [1], an example of a non-Hausdorff manifold which is not metacompact is given. We present another one.

Example 1.2. A non-Hausdorff manifold $M$ need not to be metacompact.

Let $M=\mathbb{R} \cup(\mathbb{Q} \times\{1\})$ and define a topology on $M$ as follows.

(i) For each $x \in \mathbb{R}$, a basic open neighborhood of $x$ is open in $\mathbb{R}$ with the usual topology.

(ii) For each $(q, 1) \in \mathbb{Q} \times\{1\}$, a basic open neighborhood of $(q, 1)$ is of the form $[\{(q, 1)\} \cup$ $U] \backslash\{q\}$ where $U$ is an open neighborhood of $q$ in $\mathbb{R}$ with the usual topology.

Claim 1. The non-Hausdorff manifold $M$ is not metacompact.

Proof. Let $\mathcal{U}=\{\{(q, 1)\} \cup \mathbb{R}: q \in \mathbb{Q}\}$. To see that $\mathcal{U}$ has no point finite refinement, let $\mathcal{U}$ be a refinement of $\mathcal{U}$. Let $q_{0} \in \mathbb{Q}$ and $\varepsilon_{0}>0$ such that $\left(q_{0}-\varepsilon_{0}, q_{0}+\varepsilon_{0}\right)$ is a subset of some element of $\mho$. For each $n \in \mathbb{N}$, choose $q_{n} \in \mathbb{Q}, \varepsilon_{n}>0$, and $V_{n} \in \mathcal{U}$ such that $\left[q_{n}-\varepsilon_{n}, q_{n}+\varepsilon_{n}\right] \subseteq$ $\left(q_{n-1}-\varepsilon_{n-1}, q_{n-1}+\varepsilon_{n-1}\right) \backslash\left\{q_{n-1}\right\}, \varepsilon_{n}<1 / n$, and $\left(\left\{\left(q_{n}, 1\right)\right\} \cup\left[q_{n}-\varepsilon_{n}, q_{n}+\varepsilon_{n}\right]\right) \backslash\left\{q_{n}\right\} \subseteq V_{n}$. By the way $\mathcal{U}$ is defined, no element of $U$ contains more than one element of $\mathbb{Q} \times\{1\}$. Since $\mathcal{V}$ is a refinement of $\mathcal{U}$, no element of $\boldsymbol{U}$ contains more than one element of $\mathbb{Q} \times\{1\}$. Hence, $V_{j} \neq V_{k}$ whenever $j \neq k$. By Cantor's Intersection theorem, there exists $x \in \mathbb{R}$ such that $\{x\}=$ $\bigcap_{n=1}^{\infty}\left[q_{n}-\varepsilon_{n}, q_{n}+\varepsilon_{n}\right] \subseteq \bigcap_{n=1}^{\infty} V_{n}$. Therefore, $\mho$ is not point finite.

Remark 1.3. In the above example, $[0,1]$ is compact and Hausdorff but not closed.

Remark 1.4. For each $n \in \mathbb{N}, \mathbb{R}^{n}$ is a complete metric space and $\mathbb{Q}^{n}$ is a countable dense subset of $\mathbb{R}^{n}$. Therefore, a construction similar to the one above can be used to create a non-Hausdorff manifold of dimension $n$ that is not metacompact.

\section{Compatible Apparition Points}

If a manifold $M$ of dimension $n$ is non-Hausdorff, there exist at least two points $x$ and $y$ which cannot be separated by disjoint open sets. Also, the points $x$ and $y$ cannot be contained in the same Euclidean neighborhood since Euclidean neighborhoods are Hausdorff.

Definition 2.1. Let $M$ be a non-Hausdorff manifold and let $x$ and $y$ be distinct points of $M$. Then $x$ and $y$ are compatible apparition points if there do not exist disjoint open sets $U$ and $V$ with $x \in U$ and $y \in V$. By a "set of compatible apparition points," we will mean that any pair of distinct points in the set are compatible apparition points.

Remark 2.2. Since a non-Hausdorff manifold is locally Hausdorff, then no more than one element of a set of compatible apparition points can be contained in a single Euclidean neighborhood. Hence, a set of compatible apparition points is a closed discrete set. 
Remark 2.3. Since a non-Hausdorff manifold has a countable base and each point is contained in its own Euclidean neighborhood, any set of compatible apparition points must be countable.

A non-Hausdorff manifold can have an uncountable collection of sets of compatible apparition points.

Example 2.4. Let $C$ denote the Cantor ternary set and define $X=\mathbb{R} \cup(C \times\{0\})$. Define a topology on $X$ as follows.

(i) For each $x \in \mathbb{R}$, a basic open neighborhood of $x$ is open in $\mathbb{R}$ with the usual topology.

(ii) For each $x \in C$, a basic open neighborhood of $(x, 0)$ is of the form $[(x-\varepsilon, x+\varepsilon) \cap$ $C] \times\{0\} \cup(x-\varepsilon, x+\varepsilon) \backslash C$.

Note that for each $x \in C,\{x,(x, 0)\}$ is a set of compatible apparition points. Also, note that since each $\varepsilon$ can be chosen to be rational, $X$ is second countable.

Recall that a subset of a topological space is nowhere dense if the interior of its closure is empty.

Proposition 2.5. Let $S$ be a set of compatible apparition points in a non-Hausdorff manifold M. Then $S$ is nowhere dense in $M$.

Proof. Since $S$ is closed and discrete and every element of $M$ has a Euclidean neighborhood, $S$ is the frontier of $M \backslash S$ which is open. Hence, $S$ is nowhere dense by [2, $4 \mathrm{G}$ part 2 on page 37].

Proposition 2.6. Let $M$ be an n-dimensional non-Hausdorff manifold. Suppose that $M$ contains a nonempty set $S$ of compatible apparition points. Then every continuous function from $M$ to a Hausdorff space $X$ is constant on $S$.

Proof. Suppose that $f: M \rightarrow X$ is continuous. Attempting a contradiction, suppose that $x_{1}, x_{2} \in S$ such that $f\left(x_{1}\right) \neq f\left(x_{2}\right)$. Since $X$ is Hausdorff, there are disjoint open sets $U_{1}, U_{2} \subseteq X$ such that $f\left(x_{1}\right) \in U_{1}$ and $f\left(x_{2}\right) \in U_{2}$. Then $f^{-1}\left(U_{1}\right)$ and $f^{-1}\left(U_{2}\right)$ are disjoint open subsets of $M$ with $x_{1} \in f^{-1}\left(U_{1}\right)$ and $x_{2} \in f^{-1}\left(U_{2}\right)$, a contradiction.

Theorem 2.7. In a non-Hausdorff manifold, the set of points which are not apparition points is dense.

Proof. Suppose that $M$ is a non-Hausdorff manifold. Since $M$ is locally Hausdorff, Lemma 4.2 of [3] implies that each $x \in M$ has a dense open Hausdorff neighborhood $U_{x}$. Since $M$ is Lindelöf, the cover $\left\{U_{x}\right\}_{x \in M}$ has a countable subcover $\mathcal{C}$. Since $M$ is Baire, $\cap \mathcal{C}$ is dense in $M$. Since the elements of $\mathcal{C}$ are Hausdorff, any point in $\cap \mathcal{C}$ can be separated from any other point in $M$. Therefore, $\cap \mathcal{C}$ is a dense set of nonapparition points.

\section{Acknowledgments}

The authors would like to thank the referee for numerous helpful suggestions. The referee was particularly helpful in improving Theorem 2.7. 


\section{References}

[1] P. Gartside, D. Gauld, and S. Greenwood, “Homogeneous and inhomogeneous manifolds," Proceedings of the American Mathematical Society, vol. 136, no. 9, pp. 3363-3373, 2008.

[2] S. Willard, General Topology, Dover, Mineola, NY, USA, 2004.

[3] M. Baillif and A. Gabard, "Manifolds: Hausdorffness versus homogeneity," Proceedings of the American Mathematical Society, vol. 136, no. 3, pp. 1105-1111, 2008. 


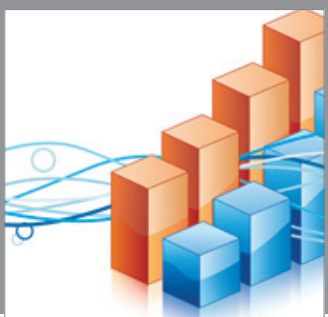

Advances in

Operations Research

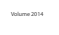

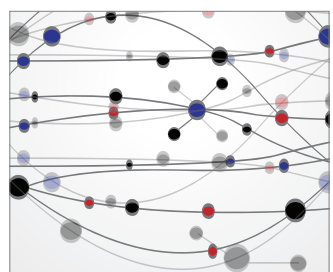

\section{The Scientific} World Journal
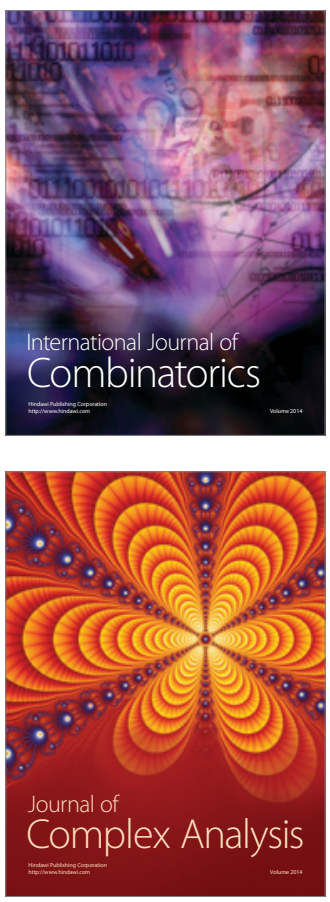

International Journal of

Mathematics and

Mathematical

Sciences
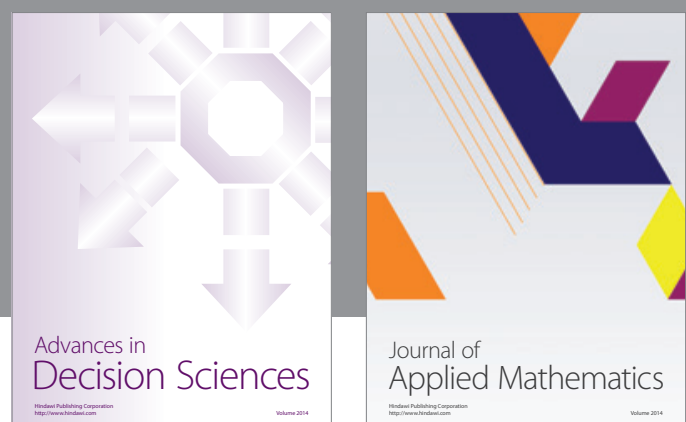

Journal of

Applied Mathematics
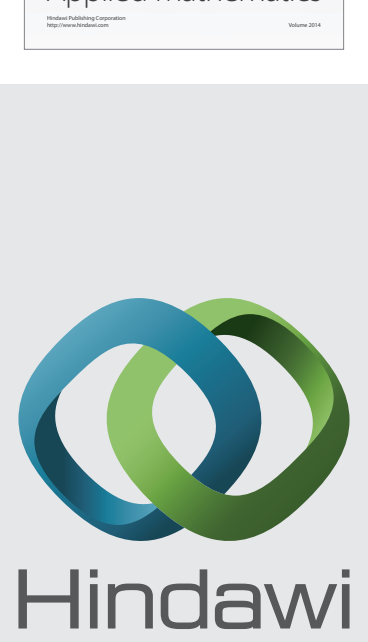

Submit your manuscripts at http://www.hindawi.com
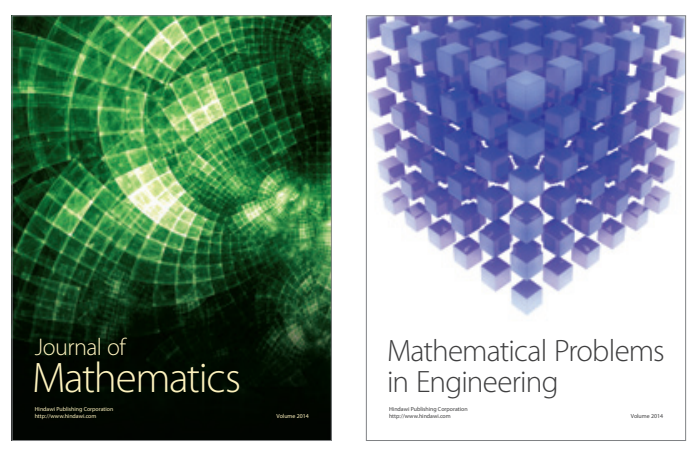

Mathematical Problems in Engineering
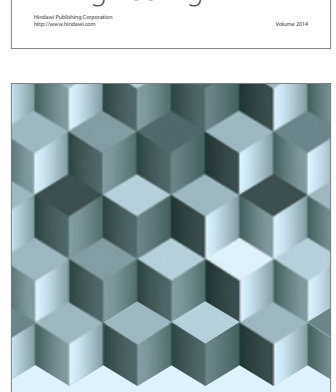

Journal of

Function Spaces
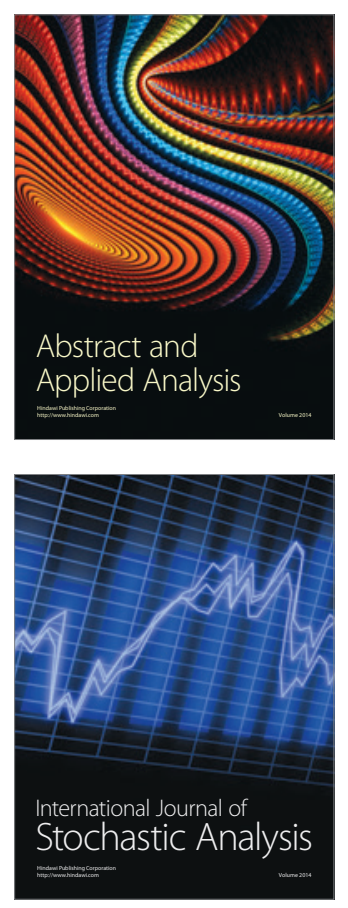

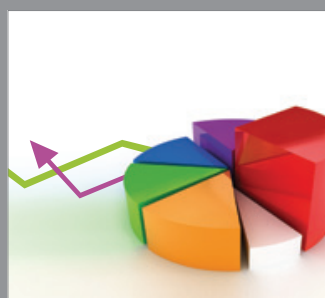

ournal of

Probability and Statistics

Promensencen
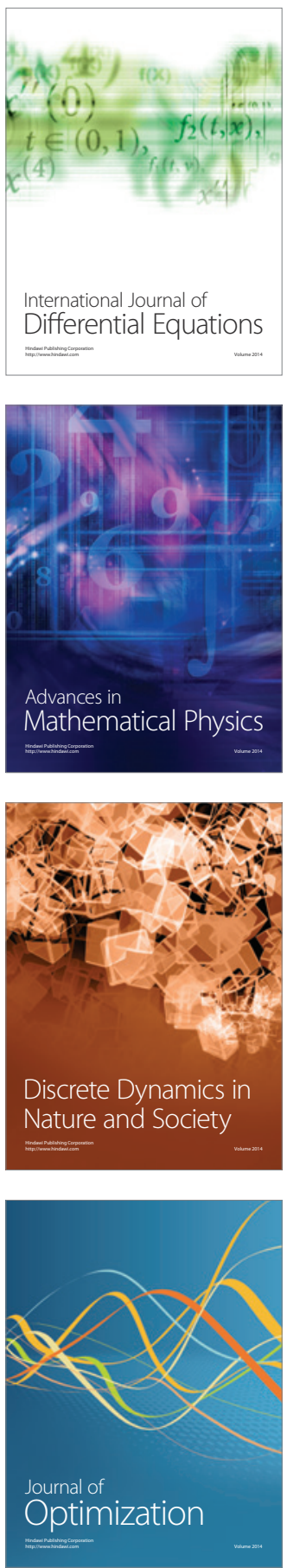